\title{
Tattua
}

JOURNAL OF PHILOSOPHY

\section{CAUSAL PROCESSES: STEPS TOWARDS A SYSTEMATIC AND FORMAL INTERPRETATION OF WHITEHEAD'S ONTOLOGY}

\author{
Ludwig Jaskolla
}

\begin{abstract}
:
Understanding the relation between Whitehead's general ontological claims and their applications in different fields of applied metaphysics has been a notorious problem since the first publication of 'Process and Reality' in 1928. The main goal of this paper is to sketch a theory of causality that is connecting with these general metaphysical claims. In order to achieve this goal, I will describe in detail one particular way in which a Whiteheadian notion of causation can be interpreted. I will spend the first part for introducing my interpretation of the general ontological principle - creativity. In the second part, I want to motivate the assertion that a theory of causality is implied by creativity. The third part will be concerned with giving a more detailed account of this theory of causality. I will argue that a Whiteheadian notion of causation is essentially bi-polar, and that there are effective and conceptual aspects to the causal role of every actual entity.
\end{abstract}




\section{Preliminary Remarks}

Understanding the relation between Whitehead's general ontological claims and their applications in different fields of applied metaphysics has been a notorious problem since the first publication of 'Process and Reality' in 1928. The main goal of this paper is to sketch a theory of causality that is connecting with these general metaphysical claims. In order to achieve this goal, I will describe in detail one particular way in which a Whiteheadian notion of causation can be interpreted. I will spend the first part of the paper giving some preliminary remarks, and introducing my interpretation of the general ontological principle - creativity. In the second part, I want to motivate the assertion that a theory of causality is implied by creativity. The third part will be concerned with giving a more detailed account of this theory of causality. I will argue that a Whiteheadian notion of causation is essentially bipolar, and that there are effective and conceptual aspects to the causal role of every actual entity. This part will be concluded by some explanatory remarks relating the Whiteheadian notion of causation to recent analytidapproaches in the theory of causality. But first let me give some hints on how I interpret the basic ontological concept of Whiteheadian philosophy - creativity. The detdiled analysis which was carried out in my paper "Causal Processes" basically relies on the following texts: Whitehead SMW, 202f, Whitehead PR, 21f, Whitehead Ai, 179-180, Whitehead RM, 98f. I argue that creativity can be adequately described as the coherent system of the sortals entailed in the category of the ultimate: Creativity as Coherence: (One $\supset \neg \diamond \neg$ Many $\wedge$ Many $\supset \neg \diamond \neg$ One)

This formal description of Creativity describes the category of the ultimate in the following way: if the concept 'One' is part of the category of the ultimate it has to hold true necessarily that it is impossible for the concept 'Many' not to be part of the category of the ultimate. Therefore, we are to conclude that Creativity describes reality to be fundamentally bi-polar.'

\section{Does Creativity Entail a Theory of Causality?}

In the following paragraphs of this paper, I want argue that the metaphysical notion of creativity entails a theory of causality. My argument is presented in a twofold manner: I will begin by clarifying the central claim that any philosophy of organism should be able to state a notion of causality. Later on, I will try to give a more

Cf. Jaskolla 2009. 
formal argument sufficing to show that the motivation given in the first part of this section does indeed hold for Whiteheadian ontology.

The philosophy of organism takes its fundamental intuitions from the experience of change and transition of the world we live in are riddled with. To emphasize this point, Whitehead quotes in a most lyrical fashion the psalmist Luke: "Abide with me / fast falls the eventide" ${ }^{\prime 2}$. The first part of the hymn expresses human craving for permanence, but the question of persistence through time and change shall not be the topic of this paper. I want to draw the readers' attention to the second part of the hymn: What Whitehead is telling us, is that despite our craving for permanence, change and transition are ultimate matters of fact governing reality. The fall of the eventide, and therefore the transition from one state of reality into another state remains a cornerstone of the philosophy of organism.

But if transition is one of the central aspects of your ontology, you better have a detailed account of how change and transition happen in the world. You also need to know the laws or the principles governing this 'How'. Therefore, you need to have a theory of how one state of reality can bring about another state of reality you need to have a theory of causality. This line of thought led me to the idea that every philosophy putting change at the very fundament of its ontological framework, needs to give a detailed account of causality.

I am going to use the rest of this section to show that one can give an argument showing the basic metaphysical principle in Whiteheadian ontology does indeed entail a theory of causality. I have called this train of thought the 'argument from adequacy' because it applies the metaontological claim for adequacy of metaphysical theories with experience of everyday life to the basic ontological concept of creativity.

On the one hand, the goal of this argument is to show that there are no explanatory gaps in Whiteheadian ontology concerning the transition from the basic ontological concepts to concrete causal processes structuring our everyday world. But on the other hand, assuming my conclusion to be valid, the argument allows for the application of certain features of creativity to a Whiteheadian notion of causality (which I will make use of in the following sections of this paper). Many scholars have reasoned that these two issues are of little importance to Whiteheadian philosophy. In particular, William Christian argued that the ontological concepts introduced in 'Process and Reality' are merely "pre-systematically"3. Apart from the fact that Christian is not able to provide a clear-cut criterion that would enable

Luke 24,29.

3. Christion 1959, 10-12. 
us to distinguish when Whitehead is talking systematically and when he is not ${ }^{4}$, it seems rather strange to develop an ontology but not to talk about anything of ontological relevance at the most basic level of this ontology. Others have argued that Whiteheadian ontology is utterly inconsistent $t^{5}$, and should consequently be used as a collection of aphorisms $s^{6}$. The problem these approaches need to cope with is in fact that Whitehead himself states clearly that providing an ontology for the philosophy of organisms is his central goal?. Therefore, if we want to follow the critical scholars cited above, we need very strong reasons supporting this kind of re-interpretation of Whiteheadian philosophy. I do not see such strong reasons given. In fact, I believe that a systematic and formal interpretation of Whiteheadian ontology in realistic terms can be given.

Nevertheless, here is the 'argument from adequacy':

01. If creativity is the basic ontological principle of Whiteheadian ontology, THEN the system of working hypothesis that can be deduced from creativity should describe reality adequately.

02. Creativity is the basic notion of Whiteheadian ontology. (cf. section 1 of this paper)

03. The system of working hypothesis that can be deduced from creativity should describe reality adequately. (1. and 2.)

04. A description of reality is adequate iff. This description accounts for every experiential structure of reality. (Cf. The definition of adequacy ${ }^{8}$. Particularly, I hold that $3 . \equiv 4$.)

05. That means: The system of working hypothesis that can be deduced from creativity accounts for every experiential structure of reality. (3. and 4.)

06. There are states of reality that are concrete, and these states of reality bear concreteness as essential part of their experiential structure. (I take this fact to be rock-bottom consensus about the ontological features of reality)

07. For every two concrete states of reality, IF these two states differ, THEN these states differ in their causal role.

08. The concept of having a certain causal role is an essential aspect of the experiential structure of concreteness. (Corollary from 7.)

\footnotetext{
Cf. Garland 1984, 214.

Cf. Buchier 1983, 282-293.

Cf. Siebers 2002, 40.

Cf. Whitehead PR, 18-19.

Cf. Whitehead PR, 4.
} 
09. That means: The system of working hypothesis that can be deduced from creativity accounts in particular for the concept of having a certain causal role. (5. and 8 .)

10. Every truth about the system of working hypothesis can (at least in principle) be deduced from creativity? (I hold that this follows from the introduction of speculative philosophy. I will give a more detailed explanation of this principle in the following paragraphs of this section.)

11. That means: The concept of having a certain causal role can (at least in principle) be deduced from creativity. OR: Creativity entails a theory of causality. (9. and 10.)

Let me give some explanatory remarks on what I proposed in these 11 steps: Due to my own interpretation of this argument, its crucial step is $\mathrm{N}^{\circ} 10$-we need to understand the motivation for, what I like to call, the structural deducibility of every particular truth from creativity. I want to argue for 10 in three steps:

(i) In his 1928 work 'Symbolism - its meaning and effect' Whitehead is addressing the question how credibility of epistemological claims can be assured. Whitehead argues that in principle every particular proposition (Whitehead summarizes every kind of reference to the world in the concept 'symbolic reference') can fail to refer ${ }^{10}$. But does this imply that in principle every epistemological claim has to be deficient? Are we forced to embrace ontological relativism when arguing for Whiteheadian ontology? I do not think that this is the case -and here is why: Whitehead is arguing that there are some epistemological concepts that refer directly to ontological structures, because these concepts are so fundamental that we need to understand them as being ontologically relevant. That there is no real argument for this transfer, only "direct, intuitive experience" 11 does not seem to be much of problem. Most fundamental philosophical concepts stem from intuition. The problem how the truths of special metaphysical frameworks can be deduced from the basic ontological concept poses itself as a partial problem of the general epistemological problem stated in 'Symbolism': The epistemological concepts Whitehead is using to introduce the notion of creativity are such that they directly refer to ontological structures. It can now be stated that creativity (as an epistemological concept) does directly refer to creativity (as a basic ontological concept).

\footnotetext{
$9 \quad$ Cf. Whitehead PR, 3-18.

1) Cf. Whitehead 1928, 7.8.

1 Garland 1983, 214.
} 
(ii) The second step accounts for the relation of Whitehead's hypothetical realism with his rationalistic belieffs ${ }^{12}$ : On the one hand, most epistemological claims are in principle deficient, despite the fact that there are some ontological structures which we are able to know to be true (what so ever). On the other hand, Whitehead's rationalism tells us that human reason is able to grasp all of reality: Combining these two positions amounts to stating that although we may never be able to formulate a perfect theory of reality as a whole, we have to admit that reality is harmonious in such way that in principle nothing is disclosed from human reason. This is what I want to define to be structural deducibility.

(iii) Whitehead's ontology can be described as monistic, because there is exactly one fundamental ontological principle -creativity. There are several hints in 'Process and Reality' underlining this point (maybe most prominently'3). At the end of the day, this amounts to the following: I argue (i) that propositions about creativity bear determinate truth values; (ii) that at least in principle every particular truth should be deducible from the basic ontological concepts; and (iii) that Whitehead's ontology is monistic. Summing up, I am able to state that structural deducibility of particulars truths from creativity is assured. In the following paragraphs, I want to sketch a Whiteheadian theory of causality.

\section{A Whiteheadian Theory of Causality}

'Process and Reality' is riddled with causal talk: Whitehead is talking about the causal powers of low-level actual entities, of higher-level actual entities (like animals or humans) and of the eternal actual entity (God). Due to the length of this paper, I will be focusing my attention to the causal powers of low-level actual entities, which means sticking to the most basic concept of causality that can be found in Whitehead's ontology.

In my paper 'Causal Processes' from 2009, I argued that an actual entity can be understood as one determinate state in the dynamic process of reality which is governed by creativity. This means that actual entities, despite being determinate states of reality, inherit from the overall creative process of reality as a whole, the

12 Cl. Whitehead PR, 214.

13 Cf. Whitehead PR, 4. 
character of being essentially dynamic: They are little processes ${ }^{14}$ that come into existence, only to perish immediately after becoming fully determinate - vash-lights of determinateness in an ever-changing universe. Unfortunately, I am only able to give this intuitive introduction, but I am conûdent that it will suffice in elucidating the following interpretations. The basic kind of causal relation between two actual entities is called a simple physical feeling ${ }^{15}$.

During the first part of this section, I will sketch the essential features of these simple physical feeling. After moving on by defining the Whiteheadian concept of causality in terms of simple physical feelings in the second part, I will address some characteristic attributes of this account of causation in the third part.

(i) Like creativity, simple physical feelings are structurally bi-polar: $O n$ the one hand, they are feelings or prehensions which mean ontologically speaking that they essentially bear the characteristic of being unities. It is always possible to refer to prehensions as determinate states of reality - for example, when I am feeling the cold creeping into the room while writing this paper, because I forgot to close the window. I am able to refer to this complex feeling as one uniued state of reality. On the other hand, prehensions are tied to their environment; they are essentially contingent on features of reality surrounding them. Again, let's have a look at the example: The determinate structure of ' $m y$ feeling the cold' is dependent on the fact that it is December and I am sitting in Germany. This means that the prehension is embedded into the conceptual structure its environment.

Another feature which stems from creativity is that simple physical feelings are very short-lived, but nevertheless extended. This entails that the variation from one simple physical feeling to another simple physical feeling will be very small - reality is everchanging, but it is changing in a harmonious way. What I am talking about is the following (and again we can make use of our example): I am still sitting in a slightly colder room. The process from one state of reality to another is not of that kind that a sudden temperature change could happen such that I have the feeling of sitting in a cold room at $t$, and that I have the feeling of sitting in a sauna at the next instant $t_{2}$. That implies the causal powers of simple physical feelings to be extremely small, and only able of making 'a big difference' over a long period of time.

14 During the following paragraphs, I will give some examples relating the conceptual framework to everyday experience. On the one hand, this entails that my example are going to be anthropomorphic. Nevertheless, I do not consider these examples to be necessary - one could find case-examples for basic actual entities and their prehensions. Despite this fact, 1 set up these examples because one is able to grasp them intuitively from one's own experience.

15

Cf. Whitehead PR, 236. 
For the last paragraph of $(I)$, I want to take a closer look at the notion of prehension, and three of its characteristic attributes. Most modern philosophers may think of prehensions as dubious entities, and here is why: Prehensions are perspectives that perceive the surrounding reality; there is a certain qualitative character to each prehension indicating how it feels to be that prehension and how it perceives the world. In analogy to Thomas Nagel, we can describe prehensions as essentially being 'views from somewhere'. Moreover, these perspectives form representations of the world they live in, and evaluate these representations. Again, most modern philosophers consider this approach dubious, because one could describe it by 'panexperientialism at its best' (or worst; depending on your perspective on the topic). But nevertheless, it's very interesting to see that in the recent, analytic debate on concrete particulars, Galen Strawson argued for a theory of panexperientialist 'simples' which bear the same characteristic attributes as Whitehead's prehensions ${ }^{16}$.

(ii) Keeping these introductory remarks in mind, we can move on and give a detailed ontological description of a simple physical feeling. All different kinds of causal structures, for example the ones mentioned at the beginning of this section, are summarized in the concept of 'causal prehension'. Due to my interpretations of Whiteheadian ontology, the concepts of 'prehension' and 'causal prehension' are semantically equivalent -that means Whitehead is using 'causal prehension' only to indicate that he is talking about the causal role of prehensions. An exegesis of 'Process and Reality' seems to underline this assertion: Whenever Whitehead is talking about 'causal prehensions' (the relevant texts are: ${ }^{17}$ ) the scope of prehensions he is addressing is the range of all prehensions. In part (I), we saw that simple physical feelings are the most fundamental prehensions of Whiteheadian ontology. So, when Whitehead is stating that "a simple physical feeling is an act of causation" 18 , we are able to draw the conclusion that he is also talking about the most fundamental causal structures there are in his ontology. At this point, it seems to be unsettled whether the set of all simple physical feelings exhausts the set of all causal structures. I think there are strong reasons indicating that this is not the case, but I am not able to give a conclusive argument during the course of this paper. I am going to leave this question open to discussion; and therefore, I am going to adopt the ontologically weakest interpretation for this problem: (3.11.1): [simple physical feelings] , $\subset$ [causal prehensions]

Sentence (3.II.1) poses an extensional definition of the concept 'simple physical feeling' implying that every simple physical feeling is a causal prehension. This

\footnotetext{
16 Cf. Strawson 2009a, 57-65 and cf. Strawson 2009b, 63-84/ 204-217.

$\checkmark \quad$ Cf. Whitehead PR, 148/161-162/176/236-238/325.

18 Whitehead PR, 236.
} 
interpretation of Whiteheadian ontology is underlined by the 'ontological principle' introduced in the basic categorical framework of 'Process and Reality ${ }^{\prime 19}$, where Whitehead is arguing that only actual entities (or prehensions between actual entities) suffice for being explananda in causal relationships. Moving on from this extensional definition, we can now take a closer look at one possible description of the intensional causal structure of simple physical feelings:

(3.II.2): A actual entity via s simple physical feeling $X_{i}\left(t_{1}\right)$ represents its environment by integrating fundamental conceptual constraints and the causal constraints of its past into different causal strains of its future. The simple physical feeling ascribes to every causal strain $\lambda$ a certain qualitative intensity $i^{\lambda}$, where $i^{\lambda}$ can be measured in positive integers $i^{\lambda} \varepsilon\left[0, \ldots\left[\right.\right.$. The causal strain with the highest $i^{\lambda}$ will become the subjective aim of the simple physical feeling defining the causal constraints causing another actual entity and its simple physical feeling $X_{1}\left(t_{2}\right)$ entailed by $i^{2}$. There are two causally relevant principles involved in this process: The effective bringing-about of new prehensions provided by the overall creative process of reality and the conceptual constraints provided by the particular prehension causing another prehension.

We need to take the integration of fundamental conceptual constraints (for example: there is no possible future where it is cold and not-cold outside) with the causal constraint of the past (for example: there is no possible future where I am in Germany at $t_{1}$ and in India at the next instant $t_{2}$ ) into different causal strains representing the environment of the simple physical feeling as matter of fact. In this paper, I am only able to give an intuitive motivation for this statement: If we take a look at the phenomenal features of our own perspective, we are able to discern three fundamental elements: Human perspectives take into account their relevant past and conceptual structures -on these grounds, we form different ideas of how we could be in the future. These possible futures were called causal strains in definition (3.II.2). Despite these phenomenological considerations, it is possible to give a formal account of this concept: Lorenz Bruno Puntel gave one possible account in his 2008 'Structure and Being'20.

One short remark on this concept of perspectives: There is no necessary reason in this account to believe that perspectives need to be conscious perspectives. We could easily imagine perspectives exemplifying only un-conscious experiences bearing the features described above. At this point, we are able to discern two different

\footnotetext{
19 Whitehead PR, 24.

D Cf. Puntel 2008, 263-264.
} 
causal principles in Whiteheadian ontology: On the one hand, there is the effective bringing-about of new prehensions that is entailed by the creative process of the universe. Creativity the basic principle of Whiteheadian ontology -is an essentially dynamical principle. This means, its sortal features can be described as follows: Every process $X$ that is governed by creativity, is essentially of that kind that it causes some following process $Y$. It can be adequately described as an 'effective bringingabout', because one process causes another process that is temporally afferwards and one process creates the following-process in a strict ontological sense. That is why, Whitehead is talking about "a creative advance into novelty"21 when talking about the overall structure of reality.

On the other hand, this raw creative power is too random to describe reality adequately. The Whiteheadian concept of causality is, therefore, able to take into account conceptual constraints limiting and bounding the effective bringing-about. We need to take a closer look at definition (3.II.2) explaining how these conceptual constraints actually work. The key principle involved in the process of forming conceptual constraints is called the 'principle of intensive relevance ${ }^{\prime 22}$. The principle states that every causal strain $\lambda$ of a simple physical feeling will be evaluated by that feeling.

The overall idea seems to be that the simple physical feeling generates a quale attributing the causal strain a certain qualitative character. If we stick to our anthropomorphic example from above, the qualitative character could be described as follows: 'I like the idea of me staying at the table and working on my paper BETTER than the idea of me getting up and closing the window". This is a rather complex situation, but let us suppose that there are only these two options. There are two qualitative characters involved - one belonging to 'staying at the table' and other belonging to 'getting up'. The interesting point is that both characters are immediately compared with each other. The one is said to 'BETTER' than the other. That means that the relations between different qualitative characters define a hierarchical structure. If we describe this relation of qualitative characters in extensional terms, we can assign to each character an integer indicating its place in the hierarchy of causal strains at a certain moment. In definition (3.II.2), I called this the qualitative intensity $i^{\lambda}$. Via $i^{\lambda}$ one causal strain is chosen, which renders the causal strain conceptually causally relevant (Whitehead calls this the subjective aim of the process) for the effective bringing-about of the new simple physical feeling. A consolidated view indicates that there are two main columns on which

\footnotetext{
21 Cf. Whitehead PR, 28.

2 Cf. Whitehead PR, 148.
} 
the Whiteheadian notion of causation relies on: To every act of causation there is an effective and a conceptual aspect ${ }^{23}$. Thus, the bi-polar nature which the concept of a simple physical feeling inherited from the basic ontological principle recurs in the theory of causation.

(iii) In the last part of this section, I want to give some explanatory remarks on different characteristic attributes of this notion of causation:

Causal Powers of other Actual Entities: Let us take closer look at how in principle the causal powers of other actual entities could look like. In 'Process and Reality' Whitehead ${ }^{24}$ argued that more complex kinds of actual entities differ in their causal power from simple physical feelings only in the variations they are able to bring about. For example, the causal powers of electrons a very limited due to the fact that their prehensions a very limited perspectives of reality - the electron could to a certain extent change position in space. We can easily see the limited possibilities of these events, when considering that they are integrated in a complex and tight net of laws of nature. On the other hand, humans have very complex (conscious) perspectives allowing them to bring about great variations - I am able to decide, for example, between staying here at my desk or meeting friends for a drink. The more complex a perspective is, the more it is able to change and interact with its environment. Due to my interpretation of the texts, I see reason to believe that Whitehead can be accounted for to propose some kind of 'causal monism'.

Inherent Vagueness of Causality: In section 2-(i) of this paper, I argued that due to Whiteheadian ontology almost every symbolic reference to the world is able to fail. That is the case, because in Whiteheadian terms the presentational immediacy of the experiential object is adapted by causal efficacy to the particular perspective of the perceiver. The notion of causal efficacy itself bears consequences to the concept of causality proposed by Whitehead: The transition of causal influences from the immediate past to the current simple physical feeling essentially omits some aspects of the past in order to focus on certain aspects of the past. These omitted aspects bear causal power, and such causal powers are not taken into account, when the simple physical feeling forms its causal strains.

Bottom line, this means that there is causal input interfering with the simple physical feeling's subjective aim rendering its causal powers essentially vague ${ }^{25}$. We need to spell this out by an example: I am deciding to go out for a drink with a friend of

\footnotetext{
23 Cf. Whitehead PR, 24.

24 Cf. Whitehead PR, 161.162.

$\approx$ Cf. Whitehead PR, 176.
} 
mine not taking into account that I have not been drinking alcohol for the last six months. That is why, I am almost immediately very drunk. One can easily see how the past interferes with my goal to have a nice evening with my friend. On the other hand, also the decisions of other simple physical feelings interfere with the subjective aims of the original simple physical feeling. Once more, I am deciding to go out for a drink with a friend of mine but in that case not taking into account she has an important exam the next day. This is why she needs reject my invitation. And again the realization of my subjective aim to have a nice evening with my friend has been severely altered.

Does this imply that like Whitehead argued this creativity-based notion of causality does essentially bear the mark of vagueness? I do not believe that this is the case. The causal structure of reality seems to be vague from the point of view of the causing actual entity. But from the point of view of for example the elernal actual entity there is a perfectly definite causal structure (in my opinion, this is a sequitur from the ontological principle). This is why, I think, the Whiteheadian concept of causality can be referred to as 'epistemological inscrutable' rather 'ontological vague'.

Causal Laws and Causal Relations: To my understanding of Whitehead bridging the gap between recent analytic metaphysics and Whiteheadian ontology is one central challenge modern Whitehead scholars need to cope with. When it comes to ranking the Whiteheadian concept of causality among recent theories of causation, we face the dire problem that we need to rank Whitehead's sometimes opaque terminology among one of the most abscure areas of research in recent analytic metaphysics. This why, I am only able to sketch some of my own classifications concerning that complex topic:

Michael Tooley provides some orientation in the philosophy of causation in the course of his book 'Causation - a Realist Approach'. Tooley argues that every theory of causality needs to ask the question whether abstract causal laws or concrete causal relations between states of realify are prior ${ }^{26}$. For the Whiteheadian concept of causality an answer seems to be at hand: The concrete, particular causal relations between different actual occasions are prior to the laws of causality. One can argue for this answer with the 'ontological principle' which states "that actual entities are the only reasons ${ }^{\prime \prime 27}$. There is just no contribution of causal powers from

x Tooley 1987, 173-175.

z Whitehead PR, 24. 
the abstract laws of nature because only the concrete actual entities are permitted as explanations in causal contexts. The inverted ontological principle can be described as follows: (3.111.1): The truths about laws of nature are logically determined by the truth-values of statements about actual entities and their causal relations ${ }^{28}$.

It is easy argue for that assertion: If (3.III.1) was not the case, there would be at least one truth about laws of nature being not logically determined solely by the truth values of statements about actual entities and their causal relations. This entails either that this particular truth would have no sufficient cause at all, or that the cause of this truth would stem from outside the realm of actual entities. The first solution occurs to me to be contra-intuitive - I consider truth without any substantiation as unintelligible. The second solution contradicts the ontological principle. Therefore, we are to conclude that (3.111.1) actually states a truth-equivalent reformulation of the ontological principle. But what are the consequences of this assertion? (3.1II.1) implies a Humean concept of laws of nature, and here is why:

Laws are universally quantified ${ }^{29}$ statements of the following kind - 'All Fs are Gs'. Basically, there are two rival approaches on laws of nature held among recent analytic metaphysicians. The first approach prominently defended by Fred Dretske, Michael Tooley and David Armstrong argues that laws of nature are contingent relations between universals: "Suppose it to be a law that Fs are Gs. F-ness and $G$-ness are taken to be universals. A certain relation, a relation of non-logical or contingent necessitation, holds between F-ness and G-ness." 30 . The second approach can be described as the humean ${ }^{31}$ concept of laws of nature and holds that what we call 'laws of nature' are merely regularities we discover while obsenving nature. ${ }^{32}$ The humean account states the laws are logically dependent upon the concrete causal relations between states of reality. Therefore, we are to conclude that when a certain causal relations changes in a way contradicting our recent set of laws of nature, we need to adjust this set. This is exactly what is implied by the ontological principle, and its inverted reformulation (3.1II.1).

20 Tooley 1987, 29.

2 At this point, it is not important whether these laws are conceived to be platonistic, humean or something in between.

30 Armstrong 1983, 85.

3 The Whiteheadian concept of causality implies a humean concept of laws, but not a humean concept of causality itself.

32 cf. for example Rosenberg 2004, 144. 


\section{Conclusive Thoughts}

In the course of this paper, I tried to give a systematic sketch of Whitehead's theory of causality. It was possible to argue that the Whiteheadian notion of causality directly stems from the basic ontological principle: The argument from adequacy shows that there are no explanatory gaps in Whiteheadian ontology concerning the concept of causality.

On the other hand, we were able to show that different features of creativity are relevant in the notion of causality. This Whiteheadian notion of causality argued that the bi-polar nature which the concept of a simple physical feeling inherited from the basic ontological principle, recurs in the theory of causation which, again, is essentially bi-polar. I think there is good reason to believe that in Whitehead's theory of causation to every causal interaction between states of reality, there are conceptual and effective causal contributions.

A more detailed analysis of the Whiteheadian concept of causality showed that one can argue for a structural causal monism in Whiteheadian ontology, as well as that despite Whitehead's own interpretation, there is no inherent vagueness entailed in this concept of causality. The comparison with recent analytic theories of causality indicated that Whitehead needs to be accounted for being a causal singularist with a humean notion of laws of nature. 